\title{
Research Article \\ Characterization of Banach Lattices in Terms of Quasi-Interior Points
}

\author{
Egor A. Alekhno \\ Faculty of Mechanics and Mathematics, Belarusian State University, 220030 Minsk, Belarus \\ Correspondence should be addressed to Egor A. Alekhno; alekhno@bsu.by
}

Received 1 April 2013; Revised 26 August 2013; Accepted 11 September 2013

Academic Editor: Gideon Schechtman

Copyright (C) 2013 Egor A. Alekhno. This is an open access article distributed under the Creative Commons Attribution License, which permits unrestricted use, distribution, and reproduction in any medium, provided the original work is properly cited.

\begin{abstract}
In terms of quasi-interior points, criteria that a Banach lattice $E$ has order continuous norm or is an $A M$-space with a unit are given. For example, if $E$ is Dedekind complete and has a weak order unit, then $E$ has order continuous norm if and only if the set of quasi-interior points of $E$ coincides with the set of weak order units of $E$; a Banach lattice $E$ is an $A M$-space with a unit $x$ if and only if the set of all quasi-interior points of $E$ coincides with the set $\{z: x \leq \lambda z$ for some $\lambda>0\}$. Analogous questions are considered for the case of an ordered Banach space $Z$ with a cone $K$. Moreover, it is shown that every nonzero point of a cone $K \neq\{0\}$ is quasi-interior if and only if $\operatorname{dim} Z=1$. We also study various $\mathscr{D}$-properties of a cone $K$; in particular, the conditions for which the relation $a \wedge b=0$ with $a>0$ implies that $b$ is not a quasi-interior point are considered.
\end{abstract}

\section{Introduction}

One of natural notions from the theory of Riesz spaces is a notion of a weak order unit: an element $x>0$ in a Riesz space $E$ is said to be [1, page 36] a weak order unit whenever for each $z \in E$, the relation $z \perp x$ implies $z=0$ (or, equivalently, whenever $z \wedge n x \uparrow z$ for each $z \geq 0$ ). A topological analog of a weak order unit is a quasi-interior point. Namely, an element $x>0$ in a Banach lattice $E$ is said to be [1, page 259] a quasiinterior point, whenever for each $z \geq 0$, the sequence $z \wedge n x$ norm converges to $z$. This is equivalent to the assertion that $x^{*} x>0$ for each nonzero positive functional $x^{*} \in E^{*}$. In the case of an ordered Banach space $Z$ with a cone $K$, the last assertion is taken up as a definition of a quasi-interior point of $K$ (see [2, page 24]).

The main idea of using quasi-interior points is the following. If an element $x$ of a Banach lattice $E$ is quasiinterior, then, on the one hand, the (order) ideal $I_{x}$ generated by $x$ is norm dense in $E$; on the other hand, the ideal $I_{x}$ under the norm $\|z\|_{x}=\min \{\lambda \geq 0:|z| \leq \lambda x\}$ is an AM-space with a unit $x$ and so that, by Kakutani-Bohnenblust-Kreins' theorem [1, page 194], $I_{x}$ is lattice isometric onto a space $C(S)$ of all continuous functions on some compact topological space $S$. Thus, it is often possible to reduce the investigation of number properties of a Banach lattice $E$ to the study of these properties, or closed to them, in the space of continuous functions, and then, since $I_{x}$ is dense in $E$, we can return to the space $E$. In other words, from all Banach lattices, Banach lattices having quasi-interior points are the "closest" to $A M$ spaces with a unit.

In spite of the considerable progress in the theory of quasi-interior points (see [3, pages 14-17] for precise and detailed reference), several aspects of this theory have received almost no attention. Up to now, conditions which give the characterization of an order structure of an arbitrary Banach lattice $E$ with help of quasi-interior points were not studied. For example, conditions in terms of quasi-interior points guaranteeing that a Banach lattice $E$ is an $A M$-space with a unit were not known. The class of Banach lattices with order continuous norms which have been researched well and are important was not even considered with the point of view of quasi-interior points. Moreover, the following question raised to Schaefer [4, page 270, Remark (iii)] were remained without an answer: Does a cone $K$ of some Banach space $Z$, $\operatorname{dim} Z>1$, such that every nonzero element $z \in K$ is a quasiinterior point of $K$, exist?

The principal purpose of this paper is to give in terms of quasi-interior points the characterizations of two most important classes of Banach lattices, namely, Banach lattices with order continuous norms and $A M$-spaces with a unit. 
The second section is devoted to these problems. In the third section, analogous questions are considered for ordered Banach spaces, and a negative answer to Schaefer's problem stated above is provided (Theorem 6). The objective of Section 4 is to introduce various $\mathscr{D}$-properties of a cone and to study them in conjunction with the notion of quasiinterior point. The last section presents some applications of the theory of ordered Banach algebras to the scope of questions discussed in the preceding sections.

For any unexplained terminology, notations, and elementary properties of Banach lattices, we refer the reader to $[1,5]$. For information on the theory of ordered Banach spaces, we suggest [2] (see also [6]). Throughout the paper, unless stated otherwise, $E$ will stand for an arbitrary Banach lattice, while $Z$ will be an arbitrary ordered Banach space with a (closed) cone $K$. We assume that $E \neq\{0\}$ or $K \neq\{0\}$.

\section{The Case of a Banach Lattice}

We start with a simple result that will be needed later for the characterization of Banach lattices, having order continuous norms with help of quasi-interior points.

Lemma 1. Let $E$ be a Banach lattice with the countable sup property and let $J$ be a closed ideal in $E$, such that $J^{d}=\{0\}$. If $E$ has a weak order unit $x$, then ideal $J$ also has a weak order unit.

Proof. From the relation $J^{\mathrm{d}}=\{0\}$, it follows that the ideal $J$ is order dense in $E$. Consequently, there exists a sequence $x_{n} \in J$ satisfying $0<x_{n} \uparrow x$. Then, the element $x_{0}=$ $\sum_{i=1}^{\infty}\left(1 / 2^{i}\left\|x_{i}\right\|\right) x_{i} \in J$ is a weak order unit (in $E$ ). Indeed, if $z \perp x_{0}$ with $z \in E$, then $|z| \wedge x_{n}=0$ for all $n$. Since $|z| \wedge x_{n} \uparrow|z| \wedge x$, we obtain $|z| \wedge x=0$ or $z=0$.

In order to proceed further, we recall the well-known fact that every Banach lattice $E$ with order continuous norm has the countable sup property.

Theorem 2. A Banach lattice $E$ with the countable sup property and with a weak order unit has order continuous norm if and only if the set of all quasi-interior points of E coincides with the set of all weak order units of $E$.

Proof. The necessity is valid without the assumption that $E$ has the countable sup property and can be found, for example, in [1, page 260]. We will prove the sufficiency. Assume by way of contradiction that $E$ does not have order continuous norm. This is equivalent to the fact that the band $E_{n}^{\sim}$ of all order continuous functionals does not coincide with the norm dual $E^{*}$ of $E$. Therefore, there exists a functional $x^{*}>0$ satisfying the relation $N_{x^{*}}^{d}=\{0\}$, where $N_{x^{*}}=\left\{z \in E: x^{*}|z|=0\right\}$. Next, $N_{x^{*}}$ is a closed ideal; hence, by Lemma $1, N_{x^{*}}$ has a weak order unit $x$. By our hypothesis, the element $x$ is a quasiinterior point. However, $x^{*} x=0$, which is impossible.

The preceding theorem is also valid if we replace the property that $E$ has the countable sup property by the Dedekind completeness of $E$ (see Theorem 14 later). Moreover, Theorem 2 can be obtained with help of the next Andô theorem [1, page 173]: a Banach lattice $E$ has order continuous norm if and only if every closed ideal is a band.

The following examples show that, in Lemma 1 and Theorem 2, the condition that $E$ has the countable sup property is essential.

Example 3. Consider the Banach lattice $B(\mathbb{R})$ of all bounded functions $z: \mathbb{R} \rightarrow \mathbb{R}$ with the norm $\|z\|=\sup _{s \in \mathbb{R}}|z(s)|$, and let $J$ be the closed ideal in $B(\mathbb{R})$ consisting from all functions which have countable supports. Then $J^{\mathrm{d}}=\{0\}$ and $J$ does not have a weak order unit.

Example 4. Let $B(\mathbb{R})$ be the space from the preceding example and let $e \in B(\mathbb{R})$ be the constant function one on $\mathbb{R}$. Through $c_{0}(\mathbb{R})$, we denote a closed subspace of $B(\mathbb{R})$ consisting from all functions $z$, such that for every $\epsilon>0$ the set $\{s:|z(s)|>\epsilon\}$ is finite. Consider the space

$$
E=\left\{i+\lambda e \in B(\mathbb{R}): i \in c_{0}(\mathbb{R}), \lambda \in \mathbb{R}\right\} .
$$

It is closed in $B(\mathbb{R})$. Actually, if a sequence $i_{n}+\lambda_{n} e \rightarrow z$ with $i_{n} \in c_{0}(\mathbb{R}), \lambda_{n} \in \mathbb{R}$, and $z \in B(\mathbb{R})$, then $\lambda_{n}$ is a bounded sequence. Consequently, we can assume that $\lambda_{n}$ converges to some $\lambda_{0}$. In this case, $z-\lambda_{0} e \in c_{0}(\mathbb{R})$. Next, $E$ is also closed under the lattice operations. Thus, $E$ is a Banach lattice. Nevertheless, $E$ does not have order continuous norm, and, on the other hand, the set of all quasi-interior points of $E$ coincides with the set of all weak order units of $E$. We mention that $E$ is an AM-space with a unit which is not Dedekind complete (see Theorem 14 and Corollary 15).

The most classical Banach lattice which does not have order continuous norm is an infinite dimensional AM-space with a unit. In the following theorem, the characterization of such spaces in terms of quasi-interior points is given. Recall that a Riesz space $F$ has [1, page 35] the principal projection property if for every element $z \in F$ the band $B_{z}$ generated by $z$ is a projection band; that is, $B_{z} \oplus B_{z}^{d}=F$ or, in other words, there exists an order projection $P_{z}$ from the space $F$ onto $B_{z}$.

Theorem 5. For a Banach lattice $E$ and an element $x \in E^{+}$, the following two statements are equivalent:

(a) $E$ is an AM-space with a unit $x$;

(b) the set of all quasi-interior points of $E$ coincides with the set of all elements $z$, such that $x \leq \lambda z$ for some $\lambda>0$.

Moreover, in case E has the principal projection property, each from the conditions $(a)$ and $(b)$ is equivalent to the next:

(c) the relation $\inf _{z \neq 0}\left\|P_{z} x\right\|>0$ holds.

Proof. First of all, we recall that an element $y$ is a unit of some Banach lattice $F$ if and only if $y$ is an interior point of $F^{+}$. On the other hand, if in some ordered Banach space $Z$ with a cone $K$ the interior $\stackrel{\circ}{K}$ of $K$ is nonempty, then it is well known (see, e.g., [2, page 22$])$ that $\stackrel{\circ}{K}$ coincides with the set of all quasi-interior points of $K$. Now, the implication (a) $\Rightarrow$ (b) 
is obvious. For a proof of (a) $\Rightarrow(\mathrm{c})$, it suffices to observe that the norms $\|\cdot\|_{x}$ and $\|\cdot\|$ are equivalent, and the identities $\left\|P_{z} x\right\|_{x}=\|x\|_{x}=1$ hold. Indeed, if $\left\|P_{z} x\right\|_{x}<1$, then $P_{z} x \leq \lambda x$ for some nonnegative scalar $\lambda<1$; hence, $P_{z} x \leq \lambda P_{z} x$ or $P_{z} x=0$. Thus, $z \perp x$, which is impossible (in the next section, this result will be stated for the case of an ordered Banach space (Theorem $5^{\prime}$ ), and in the same place another approach to a proof of the implication (a) $\Rightarrow$ (c) can be found).

(c) $\Rightarrow$ (a) Suppose by way of contradiction that $x$ is not an interior point of $E^{+}$. Then, there exists a sequence $x_{n}$ satisfying $x_{n} \rightarrow x$ and $x_{n} \notin E^{+}$. The order projections $P_{\left(x_{n}\right)^{-}}$ are nonzero. Clearly, $P_{\left(x_{n}\right)^{-}}\left(x_{n}-x\right) \rightarrow 0$. Hence, using the inequality $P_{\left(x_{n}\right)^{-}} x \leq P_{\left(x_{n}\right)^{-}} x-P_{\left(x_{n}\right)^{-}} x_{n}$, we infer $P_{\left(x_{n}\right)^{-}} x \rightarrow 0$. The latter is contrary to $(c)$.

(b) $\Rightarrow$ (a) Again suppose by way of contradiction that $x$ is not an interior point of the set $E^{+}$. This set is ideally convex; therefore [2, pages 10,11], $x$ is not an interior point of $E^{+}$if and only if $x$ is not $c$-interior point of $E^{+}$. Consequently, there exists an element $y \in E$ satisfying $x-(1 / n) y \notin E^{+}$for all $n$. Since $x-(1 / n) y^{+} \leq x-(1 / n) y$, we can assume that $y>0$. Put $x_{n}=x-(1 / n) y$. Clearly, $x_{n} \uparrow x$ and $x_{n}$ (norm) converge to $x$. Define the element $x_{0}=\sum_{i=1}^{\infty}\left(1 / 2^{i}\right)\left(x_{i}\right)^{+}$. We will establish that $x_{0}$ is a quasi-interior point. To this end, proceeding by contradiction, we find a functional $x^{*}>0$ satisfying $x^{*} x_{0}=$ 0 . Therefore, $x^{*}\left(x_{n}\right)^{+}=0$ for all $n$. Whence, using the relation

$$
\left(x_{n}\right)^{+} \longrightarrow x
$$

we get $x^{*} x=0$, which is impossible. By the condition (b), for some natural number $k$, the inequality $x \leq 2^{k} x_{0}$ holds. Taking into account the relation $P_{\left(x_{k+1}\right)^{-}}\left(x_{j}\right)^{+}=0$ for all $j=$ $1, \ldots, k+1$, we have

$$
\begin{aligned}
P_{\left(x_{k+1}\right)^{-}} x & \leq \sum_{i=1}^{\infty} \frac{2^{k}}{2^{i}} P_{\left(x_{k+1}\right)^{-}}\left(x_{i}\right)^{+} \\
& =\sum_{i=k+2}^{\infty} \frac{2^{k}}{2^{i}} P_{\left(x_{k+1}\right)^{-}}\left(x_{i}\right)^{+} \\
& \leq \sum_{i=k+2}^{\infty} \frac{2^{k}}{2^{i}} P_{\left(x_{k+1}\right)^{-}} x=\frac{1}{2} P_{\left(x_{k+1}\right)^{-}} x .
\end{aligned}
$$

Consequently, $P_{\left(x_{k+1}\right)^{-}} x=0$; hence, $x \perp\left(x_{k+1}\right)^{-}$. Finally, $\left(x_{k+1}\right)^{-}=0$ or $x_{k+1} \geq 0$, and we have a contradiction.

For the case of Banach function spaces, Theorems 2 and 5 were obtained in [7], and then in [3] they were generalized for the case of Banach lattices in some other view. Analogues of these theorems for cones can be found in the next section (Theorems $2^{\prime}$ and $5^{\prime}$, resp.).

We will close this section with the following remarks. As a matter of fact, in the proof of Theorem 5, the validity of the next assertion was shown: if $x$ is a quasi-interior point of a Banach lattice $E$, a sequence $x_{n}$ converges to $x$, and a sequence $\alpha=\left(\alpha_{1}, \alpha_{2}, \ldots\right) \in \ell_{1}$, such that $\alpha_{n} \neq 0$ for all $n$, then the element $x_{0}=\sum_{i=1}^{\infty}\left|\alpha_{i}\right|\left(x_{i}\right)^{+}$is quasi-interior. Clearly, if $\left|x_{n}\right| \leq x$, then $x_{0} \in I_{x}$. The converse statement is also true: if an element $x \in E^{+}$and an element $x_{0} \in I_{x}$ are quasi-interior points of $E$, then there exist an increasing sequence $x_{n} \geq 0$ converging to $x$ and a sequence $\alpha=\left(\alpha_{1}, \alpha_{2}, \ldots\right) \in \ell_{1}$ satisfying $\alpha_{n}>0$ for all $n$, such that $x_{0}=\sum_{i=1}^{\infty} \alpha_{i} x_{i}$. In fact, we can assume that $0<$ $x_{0} \leq x$. For natural numbers $n \geq 2$, we put $x_{n}=x \wedge\left(n x_{0}\right)$ and $\alpha_{n}=1 / 2^{n-1} n$. Then, $\sum_{i=2}^{\infty} \alpha_{i} x_{i} \leq x_{0}$. Define $x_{1}=x_{0}-\sum_{i=2}^{\infty} \alpha_{i} x_{i}$ and $\alpha_{1}=1$. It remains to observe the validity of the relations $0 \leq x_{1} \leq x_{0} \leq x_{2}$ and $x_{0}=\sum_{i=1}^{\infty} \alpha_{i} x_{i}$.

An analogous result holds for the case of weak order units in a Riesz space $F$ satisfying Axiom (OS) (recall that [5, page 54] an ordered linear space $X$ satisfies Axiom (OS) if the inequalities $0 \leq z_{n} \leq \lambda_{n} z$, where $z_{n}, z \in X$ and $\lambda=\left(\lambda_{1}, \lambda_{2}, \ldots\right) \in \ell_{1}$, imply the existence of $\left.\sup _{n} \sum_{i=1}^{n} z_{i}\right)$ : if $x$ is a weak order unit in $F$, then $x_{0} \in I_{x}$ is also a weak order unit in $F$ if and only if there exist sequences $0 \leq x_{n} \uparrow x$ and $\alpha=\left(\alpha_{1}, \alpha_{2}, \ldots\right) \in \ell_{1}$ with $\alpha_{n}>0$ for all $n$, such that $\sum_{i=1}^{n} \alpha_{i} x_{i} \uparrow_{n} x_{0}$. Obviously, every $\sigma$-Dedekind complete ordered linear space $X$ and, in particular, every ordered Banach space $Z$ with a $\sigma$-minihedral cone $K$ (see the next section) satisfy Axiom (OS).

\section{The Case of an Ordered Banach Space}

The main purpose of this section is to obtain with help of quasi-interior points the characterizations of some classes of cones in Banach spaces and, in particular, to discuss possible generalizations of Theorems 2 and 5 for the case of ordered Banach spaces.

We will show first that if a Banach space $Z$ with a cone $K$ is not one-dimensional, then there exists a nonzero element $w$ of $K$ which is not a quasi-interior point of $K$ (see also [3, Theorem 1] and [8, Proposition 7]). We need to recall the following result obtained in [9] (see also [10]). Let $M$ be a closed subset of a Banach space $Y$, and let $y \in Y \backslash M$. Let $0<r<d(y, M)<R$. Then, there exists a point $w \in M$, such that $\|w-y\| \leq R$ and $M \cap \operatorname{co}(B(y, r) \cup\{w\})=\{w\}$. Here, as usual, $d(\cdot, M)$ is the distance function, $\operatorname{co}(N)$ is the convex hull of the set $N$, and $B(y, r)$ is the closed ball centered at the point $y$ with radius $r$ (below the ball $B(0,1)$ will be simply denoted by $B$ ).

Theorem 6. Let $Z$ be a Banach space, and let $K$ be a cone in $Z$. Then, the set of quasi-interior points of $K$ coincides with the set of nonzero elements of $K$ if and only if the space $Z$ is onedimensional.

Proof. The sufficiency is obvious. We will prove the necessity. Assume by way of contradiction that the dimensional $\operatorname{dim} Z>1$. We will show first that $K$ has a nonzero boundary point $z$. To this end, we find two linearly independent elements $z_{1} \in K$ and $z_{2} \notin K$. Let

$$
\lambda_{0}=\max \left\{\lambda \in[0,1]:(1-\lambda) z_{1}+\lambda z_{2} \in K\right\} .
$$

Clearly, the nonzero point $z=\left(1-\lambda_{0}\right) z_{1}+\lambda_{0} z_{2}$ is a boundary point of $K$.

We can suppose that $\|z\|=1$. Choose an element $y$ which does not belong to the cone $K$, such that $\|z-y\|<1 / 2$. Then, $d(y, K)<1 / 2$. Fix arbitrary numbers $r$ and $R$ are satisfying the inequalities $0<r<d(y, K)<R \leq 1 / 2$. There 
exists an element $w \in K$ with the properties $\|w-y\| \leq R$ and $K \cap W=\{w\}$, where the set $W=\operatorname{co}(B(y, r) \cup\{w\})$. The element $w \neq 0$. By the classical separation theorem, there exists a nonzero functional $z^{*} \in Z^{*}$, such that $z^{*} x \geq z^{*} y$ for all $x \in K$ and $y \in W$. Clearly, $z^{*} \in K^{*}$. Moreover, $z^{*} \lambda w \geq z^{*} w$ for all $\lambda \geq 0$; hence, $z^{*} w=0$. Therefore, the element $w$ is not a quasi-interior point.

Recall that a cone $K$ in a Banach space $Z$ is called [2, page 8] solid if it contains some ball of positive radius and is called (see [6, page 4] or [2, page 9]) generating (or reproducing) if $K-K=Z$. Obviously, every solid cone is generating. A cone $K$ is called [2, page 57] minihedral if every finite order bounded from above subset $S \subset Z$ has a supremum and strongly minihedral if every order bounded from above subset $S \subset Z$ has a supremum. If an arbitrary countable order bounded from above subset $S \subset Z$ has a supremum, then $K$ is called $\sigma$-minihedral. A cone $K$ is called [2, page 37] normal if for every $y \in K$ the order interval $[0, y]=\{z \in Z: 0 \leq z \leq y\}$ is norm bounded. It can be shown that [6, page 109, Exercise 12 ] $K$ is normal if and only if the space $Z$ satisfies Axiom (OS); in particular, every $\sigma$-minihedral cone is normal. If in $Z$ every increasing and order bounded above sequence $z_{n}$ converges in norm to some element $z_{0}$, then a cone $K$ is called [2, page 47] regular; in this case, it is easy to see that $z_{0}=\sup _{n} z_{n}$. Every regular cone [2, page 48] is also normal.

An arbitrary ordered Banach space $Z$ with a cone $K$ is a Riesz space (of course, under the partial ordering induced by $K$ ) if and only if $K$ is generating and minihedral. In this case, all notions from the theory of Riesz spaces can be used for the ordered Banach space $Z$, in particular, the notion of weak order unit. Moreover, as in the case of a Banach lattice, every quasi-interior point $x$ of the cone $K$ is a weak order unit. Indeed, if $x \perp y$ with $y \in Z$, then

$$
x+|y|=|y| \vee x=0 \vee(x-|y|)+|y|
$$

whence $x=(x-|y|)^{+}$. Therefore, $(x-|y|)^{+}$is a quasi-interior point of $K$. Consequently ([2, page 112]; see also Example 10 (c) below), $x \geq|y|$, so that $|y|=0$ or $y=0$.

The following result is an analogue of Theorem 2 for the case of a space with a cone.

Theorem 2'. Let $Z$ be an ordered Banach space with a generating, minihedral, and normal cone K. Moreover, let $Z$ have a weak order unit and have the countable sup property. Then $K$ is regular if and only if the set of all quasi-interior points of $K$ coincides with the set of all weak order units of $Z$.

Proof. First of all, we mention that [5, page 145, Exercise 13(a)] the cone $K$ is generating, minihedral, and normal if and only if there exists an equivalent norm on the space $Z$, such that $Z$, under this norm, is a Banach lattice. Now, it remains to remember that an arbitrary Banach lattice $E$ has order continuous norm if and only if the cone $E^{+}$is regular and to use Theorem 2.

Remark 7. Most of the constructions using the notion of quasi-interior point, for example, the representation theory (see [5, Chapter III, sections 4-6]), approximation properties of operators on Banach lattices (see [1, section 15]), or the spectral theory of ideal irreducible operators (see [5, Chapter $\mathrm{V}$, sections 5,6$]$ ) actually require that a cone $K$ enjoys the assumptions of the preceding theorem; that is, it is generating, minihedral, and normal. As it follows from the remarks done in the proof of this theorem, these assumptions actually mean that the ordered Banach space $Z$ with the cone $K$ is a Banach lattice. The given circumstance explains the reason why the notion of quasi-interior point turns out more natural and more useful for the case of a Banach lattice.

In the preceding theorem, the assumption about the normality of the cone $K$ is unnecessary if $K$ is $\sigma$-minihedral. Moreover, the necessity also holds without the explicit assumption about the normality of $K$. The next example shows that for the validity of the sufficiency, this assumption is essential.

Example 8. Consider the space $b v_{0}$ of all real sequences $z=$ $\left(z_{1}, z_{2}, \ldots\right)$ converging to zero, such that the norm $\|z\|_{b v_{0}}=$ $\sum_{i=1}^{\infty}\left|z_{i+1}-z_{i}\right|$ is finite. Under the natural ordering, that is, for elements $y, z \in b v_{0}$, we put $y \geq z$ if $y_{n} \geq z_{n}$ for all $n, b v_{0}$ is a Riesz space; in particular, the cone $\left(b v_{0}\right)^{+}$is generating and minihedral. Indeed, for $y, z \in b v_{0}$, defining the sequence $w$ by $w_{n}=y_{n} \vee z_{n}$, we have

$$
\begin{aligned}
\|w\|_{b v_{0}} & =\sum_{i=1}^{\infty}\left|y_{i+1} \vee z_{i+1}-y_{i} \vee z_{i}\right| \\
& \leq \sum_{i=1}^{\infty}\left(\left|y_{i+1}-y_{i}\right|+\left|z_{i+1}-z_{i}\right|\right)<+\infty
\end{aligned}
$$

Thus, $w \in b v_{0}$. Now, the identity $w=y \vee z$ is obvious. Moreover, the latter means that $b v_{0}$ is a Riesz subspace of the space $s$ of all real sequences; in particular, a sequence $x \in b v_{0}$ is a weak order unit if and only if $x_{n}>0$ for all $n$. Next, it is easy to see that the cone $\left(b v_{0}\right)^{+}$is closed; hence, $b v_{0}$ is an ordered Banach space. We assert that every weak order unit $x \in b v_{0}$ is a quasi-interior point of $\left(b v_{0}\right)^{+}$. Indeed, let $x^{*} x=0$ for some nonzero positive functional $x^{*} \in\left(b v_{0}\right)^{*}$. The relations $0=x^{*} x \geq x^{*}\left(x_{n} e_{n}\right)=x_{n} x^{*} e_{n} \geq 0$ hold; hence, $x^{*} e_{n}=0$ for all $n$, where $e_{n}$ is the $n$th unit coordinate vector. Consider an arbitrary element $z \in\left(b v_{0}\right)^{+}$and define $z^{(n)}=\left(z_{1}, \ldots, z_{n}, 0,0, \ldots\right)$. Clearly, $x^{*} z^{(n)}=0$. On the other hand,

$$
\left\|z-z^{(n)}\right\|_{b v_{0}}=\left|z_{n+1}\right|+\sum_{i=n+1}^{\infty}\left|z_{i+1}-z_{i}\right| \longrightarrow 0
$$

as $n \rightarrow \infty$, whence $x^{*} z=0$. Since $z$ is arbitrary, we get $x^{*}=0$, which is impossible. Finally, in $b v_{0}$, the set of quasi-interior points coincides with the set of weak order units. Nevertheless, the cone $\left(b v_{0}\right)^{+}$is not normal and, in particular, is not regular. For this, we consider the sequence $y_{n}=\sum_{i=1}^{n}(1 /(2 i-1)) e_{2 i-1}$ in $b v_{0}$ and the element $y=$ $(1,1 / 2,1 / 3, \ldots) \in b v_{0}$ and remark that $0 \leq y_{n} \leq y$ and $\left\|y_{n}\right\|_{b v_{0}}=1+2 \sum_{i=2}^{n}(1 /(2 i-1)) \rightarrow+\infty$ as $n \rightarrow \infty$. This establishes the validity of our last claim. 
We also do the following remark. If we consider the space $b v$ of all (convergent) real sequences $z$, such that the norm $\|z\|_{b v}=\left|z_{1}\right|+\sum_{i=1}^{\infty}\left|z_{i+1}-z_{i}\right|$ is finite, then under the natural ordering, $b v$ is also an ordered Banach space and, simultaneously, a Riesz space. Moreover, $b v_{0}$ is a Riesz subspace of $b v$. Evidently, if $z \in b v_{0}$, then the inequalities $\|z\|_{b v_{0}} \leq\|z\|_{b v} \leq 2\|z\|_{b v_{0}}$ hold; in particular, the cone $(b v)^{+}$is not also normal. Nevertheless, the cone $(b v)^{+}$is solid. Indeed, it suffices to show that if $e=(1,1, \ldots)$, then $B(e, 1) \subset(b v)^{+}$. For this, if $z \in B(e, 1)$, then for arbitrary number $k \in \mathbb{N}$, we have

$$
\begin{aligned}
1 & \geq\left|1-z_{1}\right|+\sum_{i=1}^{\infty}\left|z_{i+1}-z_{i}\right| \\
& \geq\left|1-z_{1}\right|+\sum_{i=1}^{k}\left|z_{i+1}-z_{i}\right| \geq\left|1-z_{k+1}\right|
\end{aligned}
$$

hence, $z_{k+1} \geq 0$, so that $z \geq 0$.

In the case of an ordered Banach space, Theorem 5 has the next form.

Theorem $5^{\prime}$. For an ordered Banach space $Z$ with a generating and minihedral cone $K$ and an element $x \in K$, the following two statements are equivalent:

(a) $K$ is a solid cone with an interior point $x$;

(b) the set of all quasi-interior points of $K$ coincides with the set of all elements $z$, such that $x \leq \lambda z$ for some $\lambda>0$.

Moreover, in case $K$ is normal and $Z$ has the principal projection property, each from the conditions (a) and (b) is equivalent to the next:

(c) the relation $\inf _{\mathrm{z} \neq 0}\left\|P_{z} x\right\|>0$ holds.

Proof. As it follows from the remarks done in the proof of Theorem $2^{\prime}$ once more, if $K$ is generating, minihedral, and normal, then the required assertion is a consequence of Theorem 5. Nevertheless, since the statement (a) implies that $\stackrel{\circ}{K}$ coincides with the set of all quasi-interior points, it is easy to see that the implication (a) $\Rightarrow$ (b) is true for an arbitrary ordered Banach space. Next, the implications $(\mathrm{a}) \Rightarrow(\mathrm{c})$ and (b) $\Rightarrow$ (a) remain valid without the assumption about the normality of the cone $K$. Indeed, let the statement (a) hold. Assume that for some sequence $z_{n} \in Z$ with $z_{n} \neq 0$ for all $n$, we have $\left\|P_{z_{n}} x\right\| \rightarrow 0$ as $n \rightarrow \infty$. For some sufficiently large $n_{0}$, the element $\left(I-P_{z_{n_{0}}}\right) x$ is an interior point of the cone $K$. Put $y=\left(I-P_{z_{n_{0}}}\right) x-P_{z_{n_{0}}} x$. Obviously, $y \notin K$. Therefore ([2, page 112]; see also Example $10(\mathrm{c})), y^{+}=\left(I-P_{z_{n_{0}}}\right) x$ is not quasi-interior, which is impossible.

Now, we will show the validity of $(b) \Rightarrow$ (a). In the proof of the analogous implication of Theorem 5, the normality of the cone $K$ was only used for the conclusion of the convergence $\left(x_{n}\right)^{+} \rightarrow x$ (see (2)), that is, in the proof of the fact that $x_{0}$ is a quasi-interior point (we use the notations from the proof of Theorem 5). Again, if $x^{*} x_{0}=0$ for some $x^{*}>0$, then $x^{*}\left(x_{n}\right)^{+}=0$ for all $n$, whence $x^{*} x_{n}=-x^{*}\left(x_{n}\right)^{-} \leq 0$. Consequently, $x^{*} x=0$, which is impossible. Finally, $x_{0}$ is a quasi-interior point.

The author is grateful to the referee for an improvement of this theorem and other useful remarks and suggestions.

It is not known whether the implication (b) $\Rightarrow(\mathrm{a})$ of the preceding theorem is valid for an arbitrary ordered Banach space and the implication (c) $\Rightarrow$ (a) is valid without the assumption about the normality of the cone $K$. Moreover, the author does not know any example of an ordered Banach space $Z$ with a cone $K$, such that $Z$ is a Riesz space with the principal projection property, while $K$ is not normal. Nevertheless, if the cone $K$ is $\sigma$-minihedral, then the implication (c) $\Rightarrow$ (a) remains valid without the assumption about the normality of $K$.

\section{Various $\mathscr{D}$-Properties of a Cone}

Let $Z$ be an arbitrary ordered Banach space with a cone $K$, and let a set $\mathscr{D} \subseteq K \times K$. A cone $K$ is said to have the weak $\mathscr{D}$ property if for every pair $(a, b) \in \mathscr{D}$ with $a>0$ there exists a functional $x^{*}>0$ satisfying $x^{*} b=0$. We mention at once the following simple result: $K$ has the weak $\mathscr{D}$-property if and only if the relations $(a, b) \in \mathscr{D}$ and $a>0$ imply that $b$ is not a quasiinterior point of $K$. A cone $K$ is said to have the $\mathscr{D}$-property if for every pair $(a, b) \in \mathscr{D}$ with $a>0$ there exists a functional $x^{*} \geq 0$ satisfying $x^{*} a>0$ and $x^{*} b=0$. Observe that if $K$ has the $\mathscr{D}$-property, then for every pair $(a, b) \in \mathscr{D}$ with $a, b>0$, the elements $a$ and $b$ are linearly independent. Next, a cone $K$ is said to have the strong $\mathscr{D}$-property if for every pair $(a, b) \in \mathscr{D}$ there exists a functional $x^{*} \geq 0$ satisfying $\left\|x^{*}\right\|=1$, $x^{*} a=\|a\|$, and $x^{*} b=0$. Obviously, the strong $\mathscr{D}$-property implies the $\mathscr{D}$-property and the $\mathscr{D}$-property implies the weak D-property.

The following result characterizes cones having the weak D-property.

Theorem 9. For an ordered Banach space $Z$ with a cone $K$ and a set $\mathscr{D} \subseteq K \times K$, the following statements are equivalent:

(a) K has the weak $\mathscr{D}$-property;

(b) if a pair $(a, b) \in \mathscr{D}$, and for every nonzero element $c \in$ $Z$, there exist two sequences $c_{n} \in Z$ converging to zero and $\lambda_{n} \in \mathbb{R}$ which satisfy the inequality $c-\lambda_{n} b \leq c_{n}$ for all $n$, then $a=0$;

(c) for every pair $(a, b) \in \mathscr{D}$ with $a>0$ there exist $a$ number $\epsilon>0$ and an element $c \in Z$ satisfying the relation

$$
\left(\bigcup_{\lambda \in \mathbb{R}}(\epsilon B+\lambda b)\right) \cap(c+K)=\emptyset .
$$

Proof. (a) $\Rightarrow$ (c) Let $(a, b) \in \mathscr{D}$ with $a>0$. There exists a functional $x^{*}>0$ satisfying $x^{*} b=0$. Fix an arbitrary element $c$ enjoying $x^{*} c>0$. We claim that $c$ is required; that is, for some number $\epsilon>0$ the relation (9) holds. To see this, proceeding by contradiction, we find sequences $b_{n} \in B$, 
$\lambda_{n} \in \mathbb{R}$, and $k_{n} \in K$, such that $(1 / n) b_{n}+\lambda_{n} b=c+k_{n} \geq c$; hence, $x^{*} c \leq(1 / n) x^{*} b_{n}+\lambda_{n} x^{*} b=(1 / n) x^{*} b_{n} \rightarrow 0$ as $n \rightarrow \infty$, which is impossible.

(c) $\Rightarrow$ (a) Again let $(a, b) \in \mathscr{D}$ with $a>0$. Pick a number $\epsilon>0$ and an element $c \neq 0$ satisfying (9). The sets $\bigcup_{\lambda \in \mathbb{R}}(\epsilon B+$ $\lambda b)$ and $c+K$ are convex, and the interior of the first one is nonempty. By the classical separation theorem, there exists a nonzero functional $x^{*} \in Z^{*}$, such that $x^{*}\left(\epsilon b^{\prime}+\lambda b\right) \leq x^{*} c+$ $x^{*} k$ for all $b^{\prime} \in B, \lambda \in \mathbb{R}$, and $k \in K$. It is easy to see that $x^{*}>0$ and $x^{*} b=0$, as desired. We also remark that $\epsilon x^{*} b^{\prime} \leq$ $x^{*} c+x^{*} k$ for all $b^{\prime} \in B$ and $k \in K$; hence, $0<\epsilon\left\|x^{*}\right\| \leq$ $x^{*} c+x^{*} k$ for all $k \in K$. Thus, $x^{*} c \neq 0$.

The check of the equivalence (b) $\Leftrightarrow$ (c) is trivial.

The validity of the following theorem can be established by analogy with the preceding one. Nevertheless, in the proof given below another approach is suggested.

Theorem $9^{\prime}$. For an ordered Banach space $Z$ with a cone $K$ and a set $\mathscr{D} \subseteq K \times K$, the following statements are equivalent:

(a) K has the D-property;

(b) if a pair $(a, b) \in \mathscr{D}$ and there exist two sequences $c_{n} \in Z$ converging to zero and $\lambda_{n} \in \mathbb{R}$ which satisfy the inequality $a-\lambda_{n} b \leq c_{n}$ for all $n$, then $a=0$;

$\left(b^{\prime}\right)$ for every pair $(a, b) \in \mathscr{D}$, there exists a number $m>0$, such that the inequality $a-\lambda b \leq c$ with $\lambda \in \mathbb{R}$ and $c \in Z$ implies $\|a\| \leq m\|c\| ;$

(c) for every pair $(a, b) \in \mathscr{D}$ with $a>0$, there exists $a$ number $\epsilon>0$ satisfying the relation

$$
\left(\bigcup_{\lambda \in \mathbb{R}}(\epsilon B+\lambda b)\right) \cap(a+K)=\emptyset .
$$

Proof. (a) $\Rightarrow$ (b) Let $a-\lambda_{n} b \leq c_{n}$ with $c_{n} \rightarrow 0$ as $n \rightarrow \infty$ and $\lambda_{n} \in \mathbb{R}$, and let $a>0$. Then, for a functional $x^{*} \geq 0$ satisfying $x^{*} a>0$ and $x^{*} b=0$, we have $x^{*} a=x^{*}\left(a-\lambda_{n} b\right) \leq x^{*} c_{n} \rightarrow 0$ as $n \rightarrow \infty$, which is impossible.

(b) $\Rightarrow$ (a) Let $(a, b) \in \mathscr{D}$ with $a>0$. We can also assume that $b>0$. Then, the elements $a$ and $b$ are linearly independent. Indeed, if not then, for some scalar $\lambda>0$, we have $a=\lambda b$, and so that $a-\lambda b=0$. Therefore, according to our hypothesis, we obtain $a=0$, a contradiction. Consider the two-dimensional subspace $Z_{0}=\{\alpha a+\beta b: \alpha, \beta \in$ $\mathbb{R}\}$ of $Z$ and define a functional $z_{0}^{*}$ on $Z_{0}$ via the formula $z_{0}^{*}(\alpha a+\beta b)=\alpha$. The desired assertion will be proved if we can verify that $z_{0}^{*}$ can be extended to a positive functional $z^{*}$ on the whole space $Z$. Suppose that such extension does not exist. By the Schaefer theorem [2, page 21, Exercise 2.3], the latter is equivalent to an unboundedness below of $z_{0}^{*}$ on the set $(B+K) \cap Z_{0}$. Consequently, we can find three sequences $b_{n} \in B$ and $\alpha_{n}, \beta_{n} \in \mathbb{R}$ for which $\alpha_{n} a+\beta_{n} b \geq b_{n}$, and $\alpha_{n} \rightarrow-\infty$ as $n \rightarrow \infty$. We can assume that $\alpha_{n}<0$ for all $n$. The inequality $a+\left(\beta_{n} / \alpha_{n}\right) b \leq\left(1 / \alpha_{n}\right) b_{n}$ is valid. Obviously, $\left(1 / \alpha_{n}\right) b_{n} \rightarrow 0$ as $n \rightarrow \infty$; hence, $a=0$, which is impossible.

(b) $\Rightarrow\left(\mathrm{b}^{\prime}\right)$ Proceeding by contradiction, for some pair $(a, b) \in \mathscr{D}$, we find two sequences $\lambda_{n} \in \mathbb{R}$ and $c_{n} \in Z$ satisfying the inequalities $a-\lambda_{n} b \leq c_{n}$ and $\left\|c_{n}\right\|<(1 / n)\|a\|$.
Then, the second inequality implies $a>0$ and $c_{n} \rightarrow 0$ as $n \rightarrow \infty$, so that from the first one, we conclude $a=0$, which is impossible.

The check of the implications $\left(b^{\prime}\right) \Rightarrow(b) \Leftrightarrow(c)$ is trivial.

Observe that the condition (b) of the preceding theorem implies the condition (b) of Theorem 9 explicitly. Actually, assume that $(a, b) \in \mathscr{D}$, and for every $c \neq 0$, there exist two sequences $c_{n}$ and $\lambda_{n}$ for which $c-\lambda_{n} b \leq c_{n}$, and $c_{n} \rightarrow 0$ as $n \rightarrow \infty$. Therefore, if $a>0$, then, in particular, we can find two sequences $c_{n}^{\prime}$ and $\lambda_{n}^{\prime}$ for which $a-\lambda_{n}^{\prime} b \leq c_{n}^{\prime}$, and $c_{n}^{\prime} \rightarrow 0$ as $n \rightarrow \infty$. A glance at the condition (b) of Theorem $9^{\prime}$ guarantees that $a=0$, a contradiction.

Next, the results of Theorems 9 and $9^{\prime}$ can be applied to the case of the set $\mathscr{D}=\{(a, b)\}$ with $a, b \in K$. For example, we have the following result: for elements $a, b \in K$ with $a>0$, there exists a functional $x^{*} \geq 0$ satisfying $x^{*} a>0$ and $x^{*} b=0$ if and only if for every sequence $c_{n}$ converging to zero and $\lambda_{n} \epsilon$ $\mathbb{R}$, the relation $c_{n}+\lambda_{n} b-a \notin K$ holds for sufficiently large $n$.

In the following example, we consider another important case of a set $\mathscr{D}$.

Example 10. Let the set

$$
\mathscr{D}=\{(a, b) \in Z \times Z: a \wedge b=0\},
$$

the set of all pairs $(a, b) \in Z \times Z$, such that the infimum $a \wedge b$ exists and is equal to zero (in other words, we consider all pairs of disjoint elements in $Z$ ).

(a) For a cone $K$, the following statements are equivalent:

(i) K has the weak D-property;

(ii) if $z \in Z \backslash K$, then the element $z^{+}=z \vee 0$, if it exists, is not a quasi-interior point of $K$;

(iii) whenever $x$ is a quasi-interior point of $K$ and $z \in$ $Z$, the relation $z \wedge x=0$ implies $z=0$.

We will check first that (i) $\Rightarrow$ (ii). Evidently, $\left(z^{-}, z^{+}\right) \in \mathscr{D}$, where $z^{-}=(-z) \vee 0>0$. Therefore, for some functional $x^{*}>0$, we have $x^{*} z^{+}=0$. For the converse, let (ii) hold and let $(a, b) \in \mathscr{D}$ with $a>0$. Put $z=b-a$. Clearly,

$$
\begin{aligned}
b & =b-a \wedge b=b+((-a) \vee(-b)), \\
& =(b-a) \vee 0=z \vee 0=z^{+} ;
\end{aligned}
$$

in particular, the element $z^{+}$exists. On the other hand, if $z \in$ $K$, then $b \geq a$; hence, $a=b \wedge a=0$, which is impossible. Thus, $z \notin K$. Consequently, $z^{+}=b$ is not a quasi-interior point; that is, $K$ has the weak $\mathscr{D}$-property. The equivalence (i) $\Leftrightarrow$ (iii) is obvious.

Next, the following assertion holds.

(b) If the linear space $K-K$ is closed, then the cone $K$ has the $D$-property; in particular, every cone in a finitedimensional space and every generating cone have the D-property. 
Without loss of generality, we can assume that $K$ is generating. Let a pair $(a, b) \in \mathscr{D}$. First of all, we mention that if for an element $u \in K$ the inequality $a-\lambda b \leq u$ holds with $\lambda \in \mathbb{R}$, then $a \leq u$. Indeed, since $u \geq a-|\lambda| b$, we can assume that $\lambda=1$. Using the inequalities $a \geq a-u$ and $b \geq a-u$, we have $0 \geq a-u$. Consequently, $u \geq a$. Next, suppose that for two sequences $c_{n} \in Z$ converging to zero and $\lambda_{n} \in \mathbb{R}$, the inequality $a-\lambda_{n} b \leq c_{n}$ holds. Since the cone $K$ is generating, we find two sequences $u_{n}, v_{n} \in K$ for which $c_{n}=u_{n}-v_{n}$ and $u_{n}, v_{n} \rightarrow 0$ as $n \rightarrow \infty$. Obviously, $u_{n} \geq a-\lambda_{n} b$; hence, from the above, we conclude $u_{n} \geq a$. Therefore, $a=0$. By the part (b) of the preceding theorem, $K$ has the $\mathscr{D}$-property.

From the parts (a) and (b), we at once obtain the following assertion (for the case of a generating and minihedral cone $K$, the validity of this assertion was mentioned in [2, page 112]).

(c) If a cone $K$ is generating and an element $z \in Z \backslash K$, then the element $z^{+}=z \vee 0$, if it exists, is not a quasi-interior point of $K$.

Nevertheless, it is important to observe that not every cone has the weak $\mathscr{D}$-property, and, as a consequence, not every cone has the $\mathscr{D}$-property (see Examples 12 and $12^{\prime}$ ).

We now turn our attention to the strong $\mathscr{D}$-property. A proof of the next theorem is analogous to proofs of Theorems 9 and $9^{\prime}$ and will be omitted.

Theorem 9". For an ordered Banach space $Z$ with a cone $K$ and a set $\mathscr{D} \subseteq K \times K$, the following statements are equivalent:

(a) K has the strong D-property;

(b) if a pair $(a, b) \in \mathscr{D}$, and an element $c \in Z$ and a number $\lambda \in \mathbb{R}$ satisfy the inequality $a-\lambda b \leq c$, then $\|a\| \leq$ $\|c\|$;

(c) for every pair $(a, b) \in \mathscr{D}$, with $a>0$, the relation

$$
\left(\bigcup_{\lambda \in \mathbb{R}}\left(B_{<\|a\|}+\lambda b\right)\right) \cap(a+K)=\emptyset
$$

holds, where $B_{<\|a\|}$ is the open ball centered at zero with radius $\|a\|$.

Example 11. Let $Z$ be an arbitrary ordered Banach space with a cone $K$, and let $\mathscr{D}$ be a set, such that $K \times\{0\} \subseteq \mathscr{D} \subseteq K \times K$.

(a) If $K$ has the strong $\mathscr{D}$-property, then the norm of $Z$ is monotone; in particular, $K$ is normal.

To see this, let $0 \leq a \leq c$. Since $(a, 0) \in \mathscr{D}$, from the part (b) of the preceding theorem, we infer $\|a\| \leq\|c\|$, which means the monotonicity of the norm (see also the part (c) below).

Now, let $\mathscr{D}$ defined by (11) be the set. First of all, it should be noticed that even in this case the monotonicity of the norm of a space $Z$ does not imply the strong $\mathscr{D}$-property. For instance, let $Z=C[0,1]$ with the sup norm, and let $K$ be a cone of all nonnegative and increasing functions of $Z$. Clearly, for a function $z \in K$, the identity $\|z\|=z(1)$ holds. Therefore, if $0 \leq_{K} y<_{K} z$, then $\|y\|<\|z\|$; that is, the norm of $Z$ is strictly monotone. However, we claim that the cone $K$ does not have the strong $D$-property (a more careful analysis shows that $K$ does not even have the weak $\mathscr{D}$-property (see Examples 12 and $12^{\prime}$ ), but we present here a direct proof that $K$ does not have the strong $\mathscr{D}$-property). To see this, we notice that for every function $z \in K$ with $z(0)=0$, the pair $(z, e) \in \mathscr{D}$, where $e$ is the constant function one on $[0,1]$. Now, fix $z \in K$ with $z(0)=0$ and $z(1)>0$, and suppose that $K$ has the strong $\mathscr{D}$ property. Then, there exists a functional $x^{*} \in K^{*}$ satisfying

$$
\left\|x^{*}\right\|=1, \quad x^{*} z=\|z\|, \quad x^{*} e=0 .
$$

It is well known that $x^{*}$ can be written as a difference of two functionals $x_{1}^{*}$ and $x_{2}^{*}$, such that $x_{1}^{*}$ and $x_{2}^{*}$ are positive, and $x_{1}^{*} \perp x_{2}^{*}$ under the natural ordering of the space $C[0,1]$ and the dual of this space. Then, using the first identity in (14), we have

$$
\begin{aligned}
1 & =\left\|x^{*}\right\|=\left\|x_{1}^{*}-x_{2}^{*}\right\|=\left\|\left|x_{1}^{*}-x_{2}^{*}\right|\right\|, \\
& =\left\|x_{1}^{*}+x_{2}^{*}\right\|=\left\|x_{1}^{*}\right\|+\left\|x_{2}^{*}\right\|,
\end{aligned}
$$

and using the third one, we have

$$
0=x^{*} e=x_{1}^{*} e-x_{2}^{*} e=\left\|x_{1}^{*}\right\|-\left\|x_{2}^{*}\right\|,
$$

whence $\left\|x_{1}^{*}\right\|=\left\|x_{2}^{*}\right\|=1 / 2$. Therefore, $x^{*} z=x_{1}^{*} z-x_{2}^{*} z \leq$ $x_{1}^{*} z \leq\|z\| / 2$, which contradicts the second identity in (14) (the condition $x^{*} \in K^{*}$ was not used).

In some more generality, the next assertion holds: if $E$ is an AM-space with a unit $e$ and $K$ is a cone in $E$, such that $K \subseteq E^{+}, e \in K$, and for some nonzero element $z \in K$, the infimum $z \wedge$ e exists under the ordering induced by $K$ and is equal to zero, then $K$ does not have the strong $\mathscr{D}$-property.

Nevertheless, it is well known that an arbitrary cone $K$ in some Banach space $Z$ is normal if and only if the space $Z$ admits an equivalent monotone norm. It is not known whenever, for a normal cone $K$, we can assert the existence of an equivalent norm under which $K$ has the strong $\mathscr{D}$-property.

As the following assertion shows, under some additional assumptions, the monotonicity of the norm implies the strong D-property.

(b) Suppose that the norms of Banach spaces $Z$ and $Z^{*}$ are monotone, and a cone $K$ is minihedral. Then, $K$ has the strong $\mathscr{D}$-property. In particular, if $E$ is a Banach lattice, then the cone $E^{+}$has the strong $\mathscr{D}$-property.

Before the check of the assertion (b), we will establish the next fact.

(c) For every $z \in K$, there exists a functional $x^{*} \in K^{*}$ satisfying $\left\|x^{*}\right\|=1$ and $x^{*} z=\|z\|$ if and only if the norm of $Z$ is monotone.

Indeed, if $0 \leq z \leq y$ and a functional $x^{*} \in K^{*}$ satisfies $\left\|x^{*}\right\|=1$ and $x^{*} z=\|z\|$, then $\|z\|=x^{*} z \leq x^{*} y \leq\|y\|$. For the converse, for a nonzero element $z \in K$, we consider the one-dimensional subspace $Z_{0}=\{\alpha z: \alpha \in \mathbb{R}\}$ of $Z$ and define a functional $z_{0}^{*}$ on $Z_{0}$ via the formula $z_{0}^{*}(\alpha z)=\alpha\|z\|$. Let us show that $z_{0}^{*}$ can be extended to a positive functional $z^{*}$ on the whole space $Z$ without increasing the norm. By the 
Schaefer theorem [2, page 21, Exercise 2.3], we must show that for every element $y \in(B+K) \cap Z_{0}$, the inequality $z_{0}^{*} y \geq$ -1 holds. To this end, proceeding by contradiction, we find a scalar $\alpha<-1 /\|z\|$ and an element $b \in B$ satisfying $\alpha z \geq b$, so that $-b \geq(-\alpha) z \geq 0$. Consequently, $1 \geq\|b\| \geq|\alpha|\|z\|>1$ must be the case, which is absurd.

Now, we are in a position to prove the assertion (b). Since the norm of $Z^{*}$ is monotone, $K^{*}$ is a normal cone. Therefore, by Andô theorem [2, page 40, Theorem 4.6], the cone $K$ is generating; hence, $Z$ is a Riesz space. Let a pair $(a, b) \in \mathscr{D}$; that is, $a, b \in K$ and $a \wedge b=0$. Using the assertion (c), we find $x^{*} \in K^{*}$ satisfying $\left\|x^{*}\right\|=1$ and $x^{*} a=\|a\|$. Next, there exists [1, page 17] a functional $y^{*}$, such that $0 \leq y^{*} \leq x^{*}, y^{*} a=x^{*} a$, and $y^{*} b=0$. Using the monotonicity of the norm of $Z^{*}$ once more, we infer $\left\|y^{*}\right\|=1$, as required.

Let $Z$ be an arbitrary ordered Banach space with a cone $K$, and let a set $\mathscr{D} \subseteq K \times K$. A cone $K$ is said to have the $\mathscr{D} \mathscr{D}$ property if for every pair $(a, b) \in \mathscr{D}$, there exists a functional $x^{*}>0$ satisfying $x^{*} a \cdot x^{*} b=0$. It is clear that a cone $K$ has the $\mathscr{D} \mathscr{D}$-property if and only if the relation $(a, b) \in \mathscr{D}$ implies that either $a$ or $b$ is not a quasi-interior point of $K$. Moreover, if $K$ has the weak $\mathscr{D}$-property, then $K$ has the $\mathscr{D} \mathscr{D}$-property.

Nevertheless, the next example shows that a cone $K$ cannot have the $\mathscr{D} \mathscr{D}$-property, even when the set $\mathscr{D}$ is defined by (11).

Example 12. Consider the set $Y=C_{0}[0,1]$ of all continuous functions $y$ on the segment $[0,1]$ with $y(0)=0$ which is an ordered Banach space equipped with a cone $K_{0}$ of all nonnegative and increasing functions of $Y$ and under the sup norm. Obviously, $Y$ is a closed subspace of $C[0,1]$ having codimension one. It is well known that the dual space $C^{*}[0,1]$ can be identified with the space $c a([0,1])$ of all (signed, $\sigma$ additive) measures (of bounded variation) defined on the $\sigma$ algebra of all Borel sets of $[0,1]$. From this, it can be easily obtained that the dual space $C_{0}^{*}[0,1]$ can be identified with the space $\{\mu \in c a([0,1]): \mu(\{0\})=0\}$; that is, in other words, for every functional $x^{*} \in C_{0}^{*}[0,1]$, there exists a unique measure $\mu_{x^{*}} \in c a([0,1])$ satisfying $\mu_{x^{*}}(\{0\})=0$ and $x^{*} y=\int_{0}^{1} y d \mu_{x^{*}}$ for all $y \in C_{0}[0,1]$; moreover, this representation preserves the natural order.

In the following assertion the description of the dual cone $K_{0}^{*}$ is given.

(a) The equality

$$
K_{0}^{*}=\left\{x^{*} \in C_{0}^{*}[0,1]: \mu_{x^{*}}([a, 1]) \geq 0 \forall a \in[0,1]\right\}
$$

holds.

To see this, let $x^{*} \in K_{0}^{*}$ and $a \in(0,1]$. Fix $\epsilon \in(0, a]$ and define the function

$$
y_{\epsilon}(t)= \begin{cases}0 & 0 \leq t \leq a-\epsilon \\ \epsilon^{-1}(t-(a-\epsilon)) & a-\epsilon \leq t \leq a \\ 1 & a \leq t \leq 1\end{cases}
$$

Obviously, $y_{\epsilon} \in K_{0}$. Hence, $\int_{0}^{1} y_{\epsilon} d \mu_{x^{*}}=x^{*} y_{\epsilon} \geq 0$. Letting $\epsilon \downarrow 0$ yields $\mu_{x^{*}}([a, 1]) \geq 0$. From this (remember that $\left.\mu_{x^{*}}(\{0\})=0\right)$, we also have $\mu_{x^{*}}([0,1]) \geq 0$. For the converse, assume that for a measure $\mu_{x^{*}}$ defining a functional $x^{*}$, the inequality $\mu_{x^{*}}([a, 1]) \geq 0$ is valid for all $a \in[0,1]$. Let $y \in K_{0}$. For an arbitrary partition $\tau=\left\{\tau_{0}, \tau_{1}, \ldots, \tau_{n}\right\}, n>1$, of the segment $[0,1]$, where, of course, $0=\tau_{0}<\tau_{1}<\cdots<$ $\tau_{n}=1$, we define a function $y_{\tau}$ on $[0,1]$ via the formulae $y_{\tau}(t)=y\left(\tau_{i}\right)$ with $t \in\left[\tau_{i}, \tau_{i+1}\right)$ and $i=0, \ldots, n-1$ and $y_{\tau}(1)=y\left(\tau_{n-1}\right)$. Since the function $y$ can be approximated uniformly by functions of the form $y_{\tau}$, it suffices to show that $\int_{0}^{1} y_{\tau} d \mu_{x^{*}} \geq 0$. To this end, let $A_{i}=\left[\tau_{i}, \tau_{i+1}\right)$ for $i=0, \ldots, n-$ 2 and $A_{n-1}=\left[\tau_{n-1}, \tau_{n}\right]$. Using an Abel transformation, we have

$$
\begin{aligned}
\int_{0}^{1} y_{\tau} d \mu_{x^{*}}= & \sum_{i=0}^{n-1} y\left(\tau_{i}\right) \mu_{x^{*}}\left(A_{i}\right) \\
= & y(0) \sum_{j=0}^{n-1} \mu_{x^{*}}\left(A_{j}\right) \\
& +\sum_{i=1}^{n-1}\left(\left(y\left(\tau_{i}\right)-y\left(\tau_{i-1}\right)\right) \sum_{j=i}^{n-1} \mu_{x^{*}}\left(A_{j}\right)\right) \\
= & \sum_{i=1}^{n-1}\left(y\left(\tau_{i}\right)-y\left(\tau_{i-1}\right)\right) \mu_{x^{*}}\left(\left[\tau_{i}, 1\right]\right) \geq 0
\end{aligned}
$$

as desired.

Now, we can give a characterization of quasi-interior points of $K_{0}$.

(b) A function $x \in K_{0}$ is a quasi-interior point of $K_{0}$ if and only if $x$ is strictly increasing on $[0,1]$.

Indeed, let $x$ be a quasi-interior point of $K_{0}$. Fix two points $t_{1}, t_{2} \in[0,1]$ with $t_{1}<t_{2}$ and define a functional $x^{*}$ on $C_{0}[0,1]$ by $x^{*}=\delta_{t_{2}}-\delta_{t_{1}}$; that is, $x^{*} y=y\left(t_{2}\right)-y\left(t_{1}\right)$ for all $y \in C_{0}[0,1]$. Evidently, $x^{*} \in K_{0}^{*}$, so that $x\left(t_{2}\right)-x\left(t_{1}\right)=$ $x^{*} x>0$. For the converse, let $x \in C_{0}[0,1]$ be a strictly increasing function, and let $x^{*} \in K_{0}^{*}$ be a nonzero functional. There exist numbers $a, b \in(0,1]$ and a number $\epsilon>0$, such that $a<b$, and the inequality $\mu_{x^{*}}([c, 1]) \geq \epsilon$ holds for every $c \in[a, b]$. Then, for an arbitrary partition $\tau=\left\{\tau_{0}, \tau_{1}, \ldots, \tau_{n}\right\}$ of the segment $[0,1]$ for which $\tau_{i}=a$ and $\tau_{i_{b}}=b$ for some indices $i_{a}, i_{b}=1, \ldots, n-1$, using (19), we have

$$
\begin{aligned}
\int_{0}^{1} x_{\tau} d \mu_{x^{*}} & \geq \sum_{i=i_{a}+1}^{i_{b}}\left(x\left(\tau_{i}\right)-x\left(\tau_{i-1}\right)\right) \mu_{x^{*}}\left(\left[\tau_{i}, 1\right]\right) \\
& \geq \epsilon \sum_{i=i_{a}+1}^{i_{b}}\left(x\left(\tau_{i}\right)-x\left(\tau_{i-1}\right)\right) \\
& =\epsilon(x(b)-x(a)) .
\end{aligned}
$$

Therefore, $x^{*} x=\int_{0}^{1} x d \mu_{x^{*}} \geq \epsilon(x(b)-x(a))>0$, and the assertion (b) is proven.

Next, it is well known that an arbitrary function $z \in$ $K_{0}-K_{0}$ defines the atomless measure $\mu_{z} \in c a([0,1])$; that is, $\mu_{z}(\{t\})=0$ for each point $t \in[0,1]$, such that for all 
$a, b \in[0,1], a<b$, the relation $\mu_{z}((a, b])=z(b)-z(a)$ is valid. Conversely, every atomless measure $\mu_{z} \in c a([0,1])$ defines the function $z \in K_{0}-K_{0}$ via the formula $z(a)=\mu((0, a])$ for $a>0$ and, obviously, $\mu=\mu_{z}$. For $z \in K_{0}-K_{0}$, we put $R z=\mu_{z}$. It is easy to see that $z \geq_{K_{0}} 0$ if and only if $\mu_{z} \geq 0$. Therefore, the vector space $K_{0}-K_{0}$ with an order generated by the cone $K_{0}$ is a Riesz space, and the operator $R$ is a lattice isomorphism between $K_{0}-K_{0}$, and the band of $c a([0,1])$ consisting from all atomless measures. From this, we conclude easily that if $y, z \in K_{0}-K_{0}$ and $w$ is an infimum of $\{y, z\}$ in $K_{0}-K_{0}$, then an infimum $y \wedge z$ also exists in the ordered Banach space $C_{0}[0,1]$ with a cone $K_{0}$ and is equal to $w$; in particular, if $y, z \in K_{0}-K_{0}$ and $y \wedge z=0$ in $K_{0}-K_{0}$ then $(y, z) \in \mathscr{D}$, where $\mathscr{D}$ is defined by (11); that is, $\mathscr{D}=\left\{(a, b) \in Y \times Y: a \wedge_{K_{0}} b=0\right\}$. In fact, evidently, $w \leq_{K_{0}} y$ and $w \leq_{K_{0}} z$. If for some element $v \in C_{0}[0,1]$, the inequalities $v \leq_{K_{0}} y$ and $v \leq_{K_{0}} z$ are true, then, since $v=y-(y-v) \in K_{0}-K_{0}$, we obtain $v \leq_{K_{0}} w$, as required. An analogous statement is also valid for a supremum $y \vee z$.

We are now ready to prove the following assertion.

(c) There exists a family $\left\{x_{p}\right\}_{p \in[0,1]}$ of elements of the cone $K_{0}$, such that $x_{p}$ is a quasi-interior point of $K_{0}$ for all $p$, and for any two indices $p^{\prime}$ and $p^{\prime \prime}, p^{\prime} \neq p^{\prime \prime}$, the relation $x_{p^{\prime}} \wedge x_{p^{\prime \prime}}=0$ holds; that is, the pair $\left(x_{p^{\prime}}, x_{p^{\prime \prime}}\right) \in \mathscr{D}$.

To see this, note first that [11, section 25] there exists a family $\left\{\mu_{p}\right\}_{p \in[0,1]}$ in $c a([0,1])$ of probability and atomless measures, such that for any two indices $p^{\prime}$ and $p^{\prime \prime}, p^{\prime} \neq p^{\prime \prime}$, we have $\mu_{p^{\prime}} \perp \mu_{p^{\prime \prime}}$ in the Riesz space $c a([0,1])$; moreover, $\mu_{p}((a, b])>0$ for all $a, b \in[0,1], a<b$, and any index $p$ (we can also assume that $\mu_{1 / 2}$ is a Lebesgue measure). Now, a required family $\left\{x_{p}\right\}$ is obtained if we put $x_{p}=R^{-1} \mu_{p}$.

The next assertion at once follows from the part (c).

(d) The cone $K_{0}$ does not have the DD-property; in particular, $K_{0}$ does not have the weak $\mathscr{D}$-property.

Example $12^{\prime}$. Consider the Banach space $Z=C[0,1]$ equipped with the cone $K$ of all nonnegative and increasing functions on $[0,1]$ (see Example 11) and the set $\mathscr{D}$ defined by the relation (11). The proofs of the following assertions are analogous to the proofs of the assertions (a) and (b) of the preceding example (again we identify functionals $x^{*} \epsilon$ $C^{*}[0,1]$ with respective measures $\left.\mu_{x^{*}} \in c a([0,1])\right)$.

(a) The equality

$K^{*}=\left\{x^{*} \in C^{*}[0,1]: \mu_{x^{*}}([a, 1]) \geq 0 \forall a \in[0,1]\right\}$

holds.

(b) A function $x \in K$ is a quasi-interior point of $K$ if and only if $x$ is strictly increasing on $[0,1]$ and $x(0)>0$.

Nevertheless, from (b), we at once obtain the next result.

(c) The cone K has the $\mathscr{D} \mathscr{D}$-property.

Indeed, if $x$ and $y$ are quasi-interior points of $K$, then $x, y \geq_{K} \min \{x(0), y(0)\} e>_{K} 0$. Hence, $(x, y) \notin \mathscr{D}$.
On the other hand, as it was mentioned in Example 11, the cone $K$ does not have the strong $\mathscr{D}$-property. In reality, we can assert more.

(d) The cone $K$ does not have the weak $\mathscr{D}$-property.

The validity of this result can be easily checked by the use of the assertion (c) of the preceding example. To see this, let $x_{p^{\prime}}$ and $x_{p^{\prime \prime}}$ be two (strictly increasing) functions from this assertion, and assume that $p^{\prime} \neq p^{\prime \prime}$. Clearly, $x_{p^{\prime}}, x_{p^{\prime \prime}} \in K$. We claim that $x_{p^{\prime}} \wedge x_{p^{\prime \prime}}=0$, where the infimum is taken in the space $C[0,1]$ with the cone $K$. To this end, note first that if $z \leq_{K} x$ and $x(0)=0$, then $z-z(0) e \leq_{K} x$. In fact, since the nonnegative function $x-z$ is increasing, we have $x(t)-z(t) \geq$ $-z(0)$ for all $t \in[0,1]$, so that the increasing function $x-$ $z+z(0) e$ is nonnegative. Now, assume that for some function $z \in Z$, the inequalities $z \leq_{K} x_{p^{\prime}}$ and $z \leq_{K} x_{p^{\prime \prime}}$ hold. By the above, we have $z-z(0) e \leq_{K} x_{p^{\prime}}$ and $z-z(0) e \leq_{K} x_{p^{\prime \prime}}$. Since $z-z(0) e \in C_{0}[0,1]$, we obtain $z-z(0) e \leq_{K} 0$. Finally, $z \leq_{K} 0$, as required. Next, fix an arbitrary number $c>0$, and put $x=$ $x_{p^{\prime \prime}}+c e$. Then, it follows from the part (b) of this example and the part (b) of the preceding one that $x$ is a quasi-interior point of $K$, and it is not difficult to see that $\left(x_{p^{\prime}}, x\right) \in \mathscr{D}$.

Nevertheless, two functions $x, y \in K$, such that $x$ is a quasi-interior point of $K, y>_{K} 0$, and $y \wedge_{K} x=0$ can be chosen more "explicitly." Indeed, the function $x(t)=t+c$ with a number $c>0$ and the Cantor function $y(t)$ satisfy the desired properties. To this end, first of all, we mention that for every functions $z_{1}, z_{2} \in Z$ and numbers $t_{1}, t_{2} \in[0,1]$, the inequalities $z_{1} \leq_{K} z_{2}$ and $t_{1} \leq t_{2}$ imply $z_{1}\left(t_{2}\right)-z_{1}\left(t_{1}\right) \leq$ $z_{2}\left(t_{2}\right)-z_{2}\left(t_{1}\right)$. Next, let $J_{n, 1}, \ldots, J_{n, 2^{n-1}}$ be the intervals deleted from the segment $[0,1]$ on the $n$th step of the construction of the Cantor set. Put $J_{n, k}=\left(a_{n, k}, b_{n, k}\right)$, where $k=1, \ldots, 2^{n-1}$. Now, we suppose that the function $z \in Z$ satisfies

$$
z \leq_{K} y, \quad z \leq_{K} x
$$

Obviously, $z(0) \leq y(0)=0$. Since $y$ is constant on the segments $\left[a_{n, k}, b_{n, k}\right]$, from the remarks done above, the function $z$ is decreasing on these segments. Consider two points $s_{1}$ and $s_{2}$ belonging to the closure of two different intervals of the form $J_{n, k}$, and show that if $s_{1}<s_{2}$, then $z\left(s_{1}\right) \geq z\left(s_{2}\right)$. We can assume without loss of generality that $s_{1}=b_{n^{\prime}, k^{\prime}}<a_{n^{\prime \prime}, k^{\prime \prime}}=s_{2}$. Fix a number $\epsilon>0$. Let $J_{i}=\left(a_{i}, b_{i}\right)$ with $i=1, \ldots, m$ be an arbitrary collection of intervals from the set $\left\{J_{n, k}\right\}$, such that the inclusion $J_{i} \subseteq\left(s_{1}, s_{2}\right)$ holds and $\sum_{i=1}^{m}\left(b_{i}-a_{i}\right)>s_{2}-s_{1}-\epsilon$. We can also assume that $b_{i}<a_{i+1}$ for all $i=1, \ldots, m-1$. In view of $(22)$, we have $z\left(b_{i}\right)-z\left(a_{i}\right) \leq 0$ for all $i=1, \ldots, m$ and $z\left(a_{i+1}\right)-z\left(b_{i}\right) \leq a_{i+1}-b_{i}$ for all $i=1, \ldots, m-1$; moreover, $z\left(a_{1}\right)-z\left(s_{1}\right) \leq a_{1}-s_{1}$ and $z\left(s_{2}\right)-z\left(b_{m}\right) \leq s_{2}-b_{m}$. Hence,

$$
\begin{aligned}
z\left(s_{2}\right)-z\left(s_{1}\right)= & z\left(s_{2}\right)-z\left(b_{m}\right)+\sum_{i=1}^{m}\left(z\left(b_{i}\right)-z\left(a_{i}\right)\right) \\
& +\sum_{i=1}^{m-1}\left(z\left(a_{i+1}\right)-z\left(b_{i}\right)\right)+z\left(a_{1}\right)-z\left(s_{1}\right)
\end{aligned}
$$




$$
\begin{aligned}
& \leq s_{2}-b_{m}+\sum_{i=1}^{m-1}\left(a_{i+1}-b_{i}\right)+a_{1}-s_{1} \\
& =s_{2}-s_{1}+\sum_{i=1}^{m}\left(a_{i}-b_{i}\right)<\epsilon .
\end{aligned}
$$

Letting $\epsilon \downarrow 0$, we obtain $z\left(s_{2}\right) \leq z\left(s_{1}\right)$. Since the set $\bigcup_{n=1}^{\infty} \bigcup_{k=1}^{2^{n-1}} J_{n, k}$ is dense in $[0,1]$, the last inequality implies the decrease of the function $z$ on this segment. Finally, $z \leq_{K} 0$, so that $y \wedge_{K} x=0$, as desired.

We close this section by the following result, showing a dependence between the $\mathscr{D} \mathscr{D}$-property and the weak $\mathscr{D}$ property. Let us denote for a set $\mathscr{D} \subseteq K \times K$ by $\mathscr{D}^{T}$ the collection $\{(a, b):(b, a) \in \mathscr{D}\}$.

Proposition 13. For an ordered Banach space $Z$ with a cone $K$ and a set $\mathscr{D} \subseteq K \times K$, the following statements are equivalent:

(a) K has the $\mathscr{D} \mathscr{D}$-property;

(b) there exists a set $\mathscr{D}_{0} \subseteq K \times K$, such that the inclusions

$$
\mathscr{D}_{0} \subseteq \mathscr{D} \cup \mathscr{D}^{T}, \quad \mathscr{D} \subseteq \mathscr{D}_{0} \cup \mathscr{D}_{0}^{T}
$$

hold, and $K$ has the weak $\mathscr{D}_{0}$-property;

(c) there exists a set $\mathscr{D}_{0} \subseteq K \times K$, such that the inclusion $\mathscr{D} \subseteq \mathscr{D}_{0} \cup \mathscr{D}_{0}^{T}$ holds, and $K$ has the weak $\mathscr{D}_{0}$-property.

Proof. Let us verify first that $(\mathrm{a}) \Rightarrow(\mathrm{b})$. Define the set $\mathscr{D}_{0}$ by the collection of all pairs $(a, b)$ such that either $(a, b) \in \mathscr{D}$ and $b$ is not a quasi-interior point of $K$ or $(b, a) \in \mathscr{D}$ and again $b$ is not a quasi-interior point of $K$. Clearly, the first inclusion in (24) holds. Next, the second one is also valid as $K$ has the $\mathscr{D} \mathscr{D}$-property. Moreover, from the construction of $\mathscr{D}_{0}$, it follows immediately that if $(a, b) \in \mathscr{D}_{0}$, then $b$ is not a quasi-interior point of $K$. Thus, $K$ has the weak $\mathscr{D}_{0}$-property. The implication (b) $\Rightarrow$ (c) is obvious. For the check of (c) $\Rightarrow$ (a), let $(a, b) \in \mathscr{D}$. We can assume that $a, b \neq 0$. Then, either $(a, b) \in \mathscr{D}_{0}$ so that $b$ is not a quasi-interior point of $K$ or $(b, a) \in \mathscr{D}_{0}$ so that $a$ is not a quasi-interior point of $K$.

We mention also that, as it is easy to see from the proof, in the preceding proposition under a more punctual choice of a set $\mathscr{D}_{0}$, the inclusion $\mathscr{D} \subseteq \mathscr{D}_{0} \cup \mathscr{D}_{0}^{T}$ can be replaced by the inclusion $\mathscr{D} \backslash((\{0\} \times K) \cup(K \times\{0\})) \subseteq \mathscr{D}_{0} \cup \mathscr{D}_{0}^{T}$.

\section{A Sight from the Point of View of Ordered Banach Algebras}

The purpose of this section is both to supplement and to make more precise results of the second section of the paper and to present another approach to this scope of questions. This approach uses some properties of the space $\mathscr{L}(E)$ of all (bounded, linear) operators on $E$ as an ordered Banach algebra.

We start by recalling that a Banach algebra $\mathscr{A}$ with a unite and with a cone $K$ is called (see, e.g., [12]) an ordered Banach algebra if $e \geq 0$, and the inequalities $y, z \geq 0$ imply $y z \geq 0$. An element $b \in \mathscr{A}$ is called [12] an order idempotent if $0 \leq b \leq e$ and $b^{2}=b$. An ordered Banach algebra $\mathscr{A}$ is said to have [12] a strongly disjunctive product if for any $y, z \geq 0$ with $y z=0$, there exists an order idempotent $b$ satisfying $y b=(e-b) z=0$.

The algebra $\mathscr{L}(E)$ under the natural algebraic operations and ordering is an ordered Banach algebra. An element $T \epsilon$ $\mathscr{L}(E)$ is an order idempotent if and only if $T$ is an order projection on $E$. It was shown in [12] that in the case of a Dedekind complete Banach lattice $E$, the algebra $\mathscr{L}(E)$ has a strongly disjunctive product if and only if $E$ has order continuous norm. This assertion implies the next result showing that Theorem 2 also remains valid if we will consider the Dedekind complete spaces instead of spaces with the countable sup property. We also recall a well-known fact that the order continuity of a norm implies the Dedekind completeness.

Theorem 14. A Dedekind complete Banach lattice $E$ with a weak order unit has order continuous norm if and only if the set of all quasi-interior points of E coincides with the set of all weak order units of $E$.

Proof. The necessity is clear (see the beginning of the proof of Theorem 2). From the remarks above, it follows that for the check of the converse assertion, it suffices to verify that $\mathscr{L}(E)$ has the strongly disjunctive product. To this end, let positive operators $S$ and $T$ on $E$ satisfy the identity $S T=0$. For a weak order unit $x \in E$, the equality $\left(I-P_{T x}\right) T x=0$ holds. Therefore,

$$
\left(I-P_{T x}\right) T=0 .
$$

Next, the element $T x+\left(I-P_{T x}\right) x$ is a weak order unit. Indeed, if $z \perp T x$ and $z \perp\left(I-P_{T x}\right) x$ then from the first relation, we obtain

$$
P_{T x} z=0
$$

and from the second one, we get $|z| \wedge\left(I-P_{T x}\right) x=0$. Hence,

$$
\begin{aligned}
0 & =\left(I-P_{T x}\right)\left(|z| \wedge\left(I-P_{T x}\right) x\right) \\
& =\left(I-P_{T x}\right)\left(\left(\left(I-P_{T x}\right)|z|\right) \wedge x\right) .
\end{aligned}
$$

Using the equality $P_{T x}\left(\left(\left(I-P_{T x}\right)|z|\right) \wedge x\right)=0$, we infer $((I-$ $\left.\left.P_{T x}\right)|z|\right) \wedge x=0$. Consequently, $\left(I-P_{T x}\right)|z|=0$. This and (26) give $z=0$. Finally, $T x+\left(I-P_{T x}\right) x$ is a quasi-interior point of $E$. Therefore, $T x$ is a quasi-interior point of $B_{T x}$. On the other hand, from $S(T x)=0$, we at once get $S P_{T x}=0$. The latter and (25) mean that the algebra $\mathscr{L}(E)$ has a strongly disjunctive product, as required.

As Example 4 shows, the assumption about the Dedekind completeness in the preceding theorem is essential. Moreover, the next result holds.

Corollary 15. Let E be a Dedekind complete AM-space with a unit. Then, the set of all quasi-interior points of E coincides with the set of all weak order units of $E$ if and only if the space $E$ is finite-dimensional. 
In the case of an ordered Banach space, Theorem 14 has the next form.

Theorem 14'. Let $Z$ be an ordered Banach space with a generating and strongly minihedral cone $K$. Moreover, let $Z$ have a weak order unit. Then, $K$ is regular if and only if the set of all quasi-interior points of $K$ coincides with the set of all weak order units of $Z$.

\section{Acknowledgments}

The main idea of the proof of Theorem 6, namely, the result from [10] used in the proof of this theorem, was suggested to the author by Professor P. P. Zabreiko. On the other hand, the existence of the family of measures $\left\{\mu_{p}\right\}$ with the properties cited in Examples 12 and $12^{\prime}$ was suggested to the author by Professor V. I. Bakhtin. The author is grateful to them for these observations.

\section{References}

[1] C. D. Aliprantis and O. Burkinshaw, Positive Operators, vol. 119 of Pure and Applied Mathematics, Academic Press, Orlando, Fla, USA, 1985.

[2] M. A. Krasnosel'skij, Je. A. Lifshits, and A. V. Sobolev, Positive Linear Systems: The Method of Positive Operators, vol. 5 of Sigma Series in Applied Mathematics, Heldermann, Berlin, Germany, 1989.

[3] E. A. Alekhno, Positive operators on ideal spaces [Ph.D. Dissertation], Belarussian State University, Minsk, Belarus, 2004.

[4] H. H. Schaefer, Topological Vector Spaces, vol. 3 of Graduate Texts in Mathematics, Springer, New York, NY, USA, 1971.

[5] H. H. Schaefer, Banach Lattices and Positive Operators, vol. 215 of Die Grundlehren der mathematischen Wissenschaften, Springer, New York, NY, USA, 1974.

[6] C. D. Aliprantis and R. Tourky, Cones and Duality, vol. 84 of Graduate Studies in Mathematics, American Mathematical Society, Providence, RI, USA, 2007.

[7] E. A. Alekhno and P. P. Zabrěko, "Quasi-positive elements and non-decomposable operators in ideal spaces. I," Proceedings of the National Academy of Sciences of Belarus. Physics and Mathematics Series, no. 4, pp. 5-9, 2002 (Russian).

[8] V. Katsikis and I. A. Polyrakis, "Positive bases in ordered subspaces with the Riesz decomposition property," Studia Mathematica, vol. 174, no. 3, pp. 233-253, 2006.

[9] H. Brézis and F. E. Browder, "A general principle on ordered sets in nonlinear functional analysis," Advances in Mathematics, vol. 21, no. 3, pp. 355-364, 1976.

[10] P. P. Zabreiko and M. A. Krasnosel'skij, "The solvability of nonlinear operator equations," Funkcional'nyi Analiz $i$ ego Priloženija, vol. 5, no. 3, pp. 42-44, 1971, translation in Russian Functional Analysis and Its Applications, vol. 5, pp. 206-220, 1971.

[11] K. R. Parthasarathy, Introduction to Probability and Measure, Springer, New York, NY, USA, 1978.

[12] E. A. Alekhno, "The irreducibility in ordered Banach algebras," Positivity, vol. 16, no. 1, pp. 143-176, 2012. 


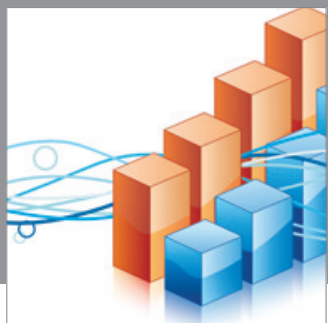

Advances in

Operations Research

mansans

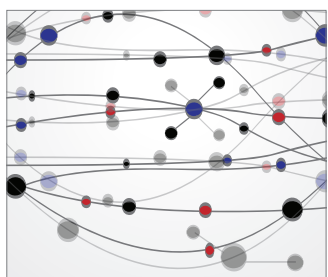

The Scientific World Journal
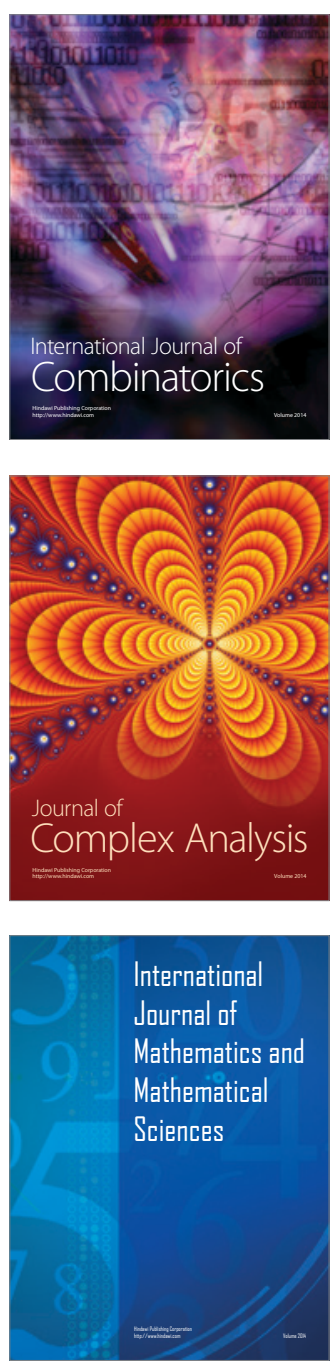
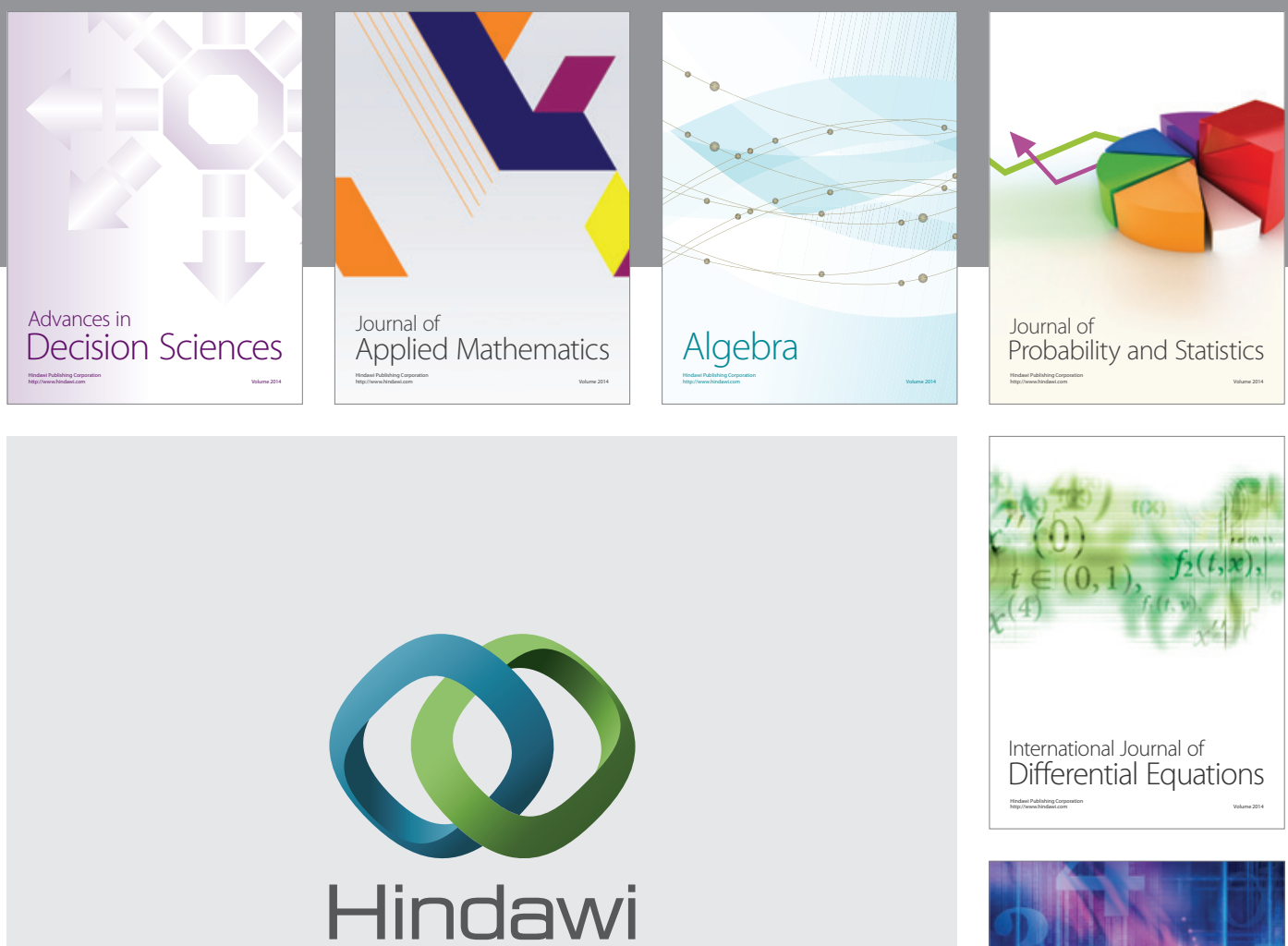

Submit your manuscripts at http://www.hindawi.com
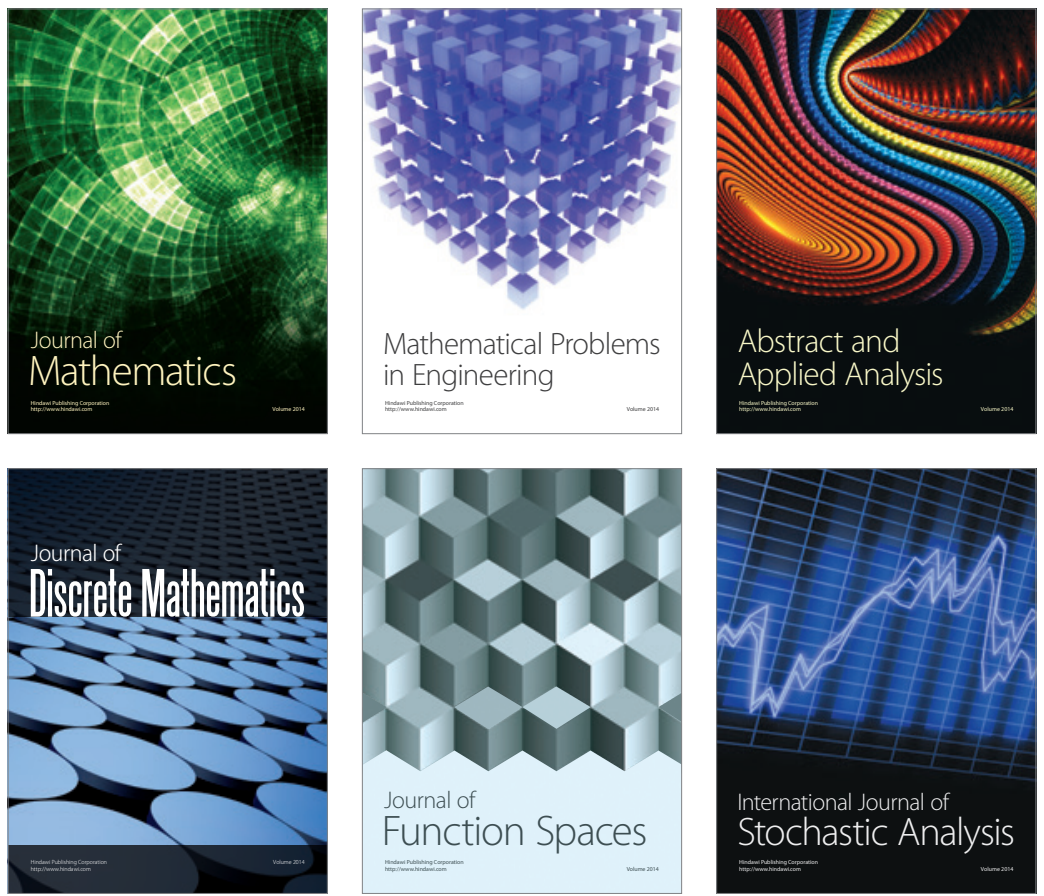

Journal of

Function Spaces

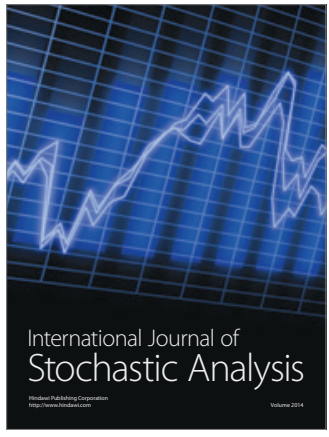

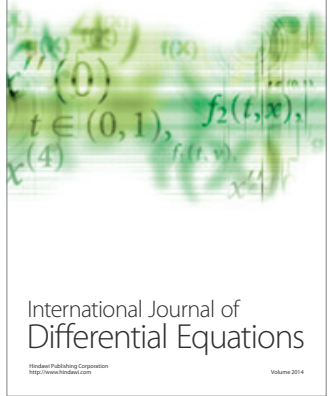
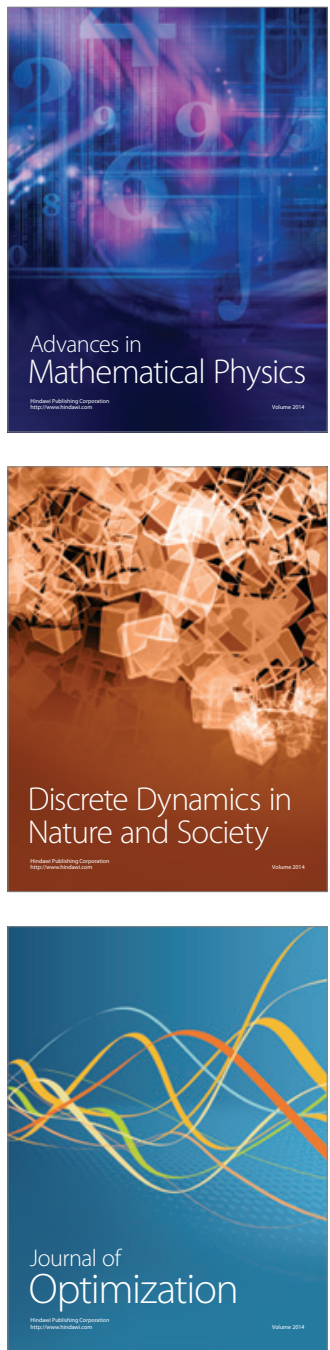